\title{
Un fil d'Ariane dans le Dendi
}

Ethnographie d'une technique disparue

Ariane's Thread in the Dendi

Lucie Smolderen et Romain Minguet

\section{(2) OpenEdition}

Journals

Édition électronique

URL : https://journals.openedition.org/tc/7341

DOI : $10.4000 /$ tc. 7341

ISSN : 1952-420X

Éditeur

Éditions de l'EHESS

Édition imprimée

Date de publication : 15 décembre 2013

Pagination : 304-317

ISBN : 978-2-7351-1654-6

ISSN : 0248-6016

Référence électronique

Lucie Smolderen et Romain Minguet, « Un fil d'Ariane dans le Dendi », Techniques \& Culture [En ligne],

61 | 2013, mis en ligne le 15 décembre 2016, consulté le 29 septembre 2022. URL : http://

journals.openedition.org/tc/7341 ; DOI : https://doi.org/10.4000/tc.7341 


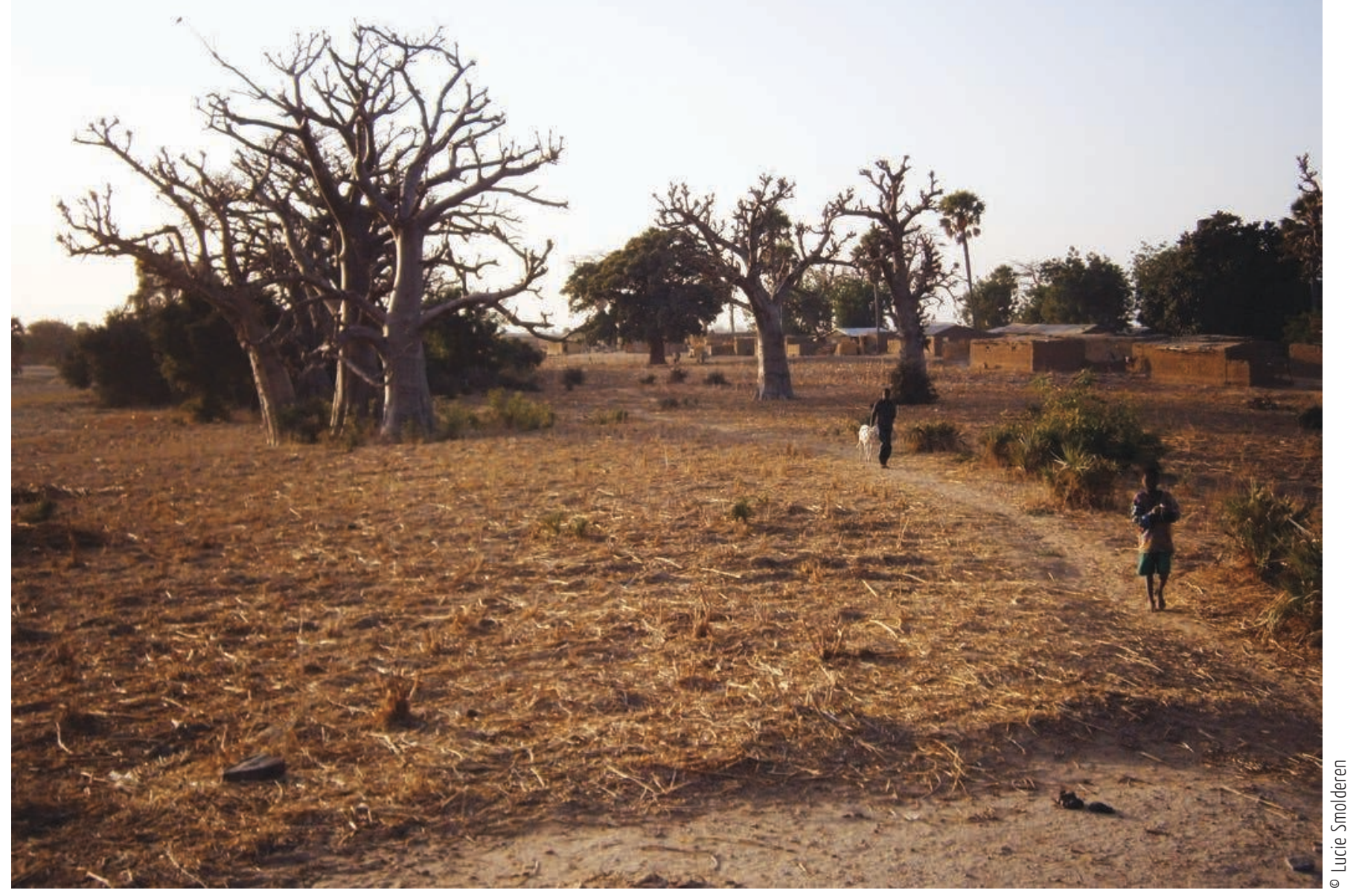




\section{UN FIL D'ARIANE DANS LE DENDI (NORD BÉNIN)}

\section{Ethnographie d'une technique disparue}

En 2012, nous entreprenons une étude technologique de la production textile artisanale du Dendi, une région du nord du Bénin — approche peu classique — . Cette filière de production n'est plus active depuis les années 1980. La colonisation, dans un premier temps, (et l'appropriation de la production de coton par les Français), l'Indépendance, dans un second, (avec la mise en place progressive d'une politique d'exportation) ont eu raison de la production textile artisanale. En une génération seulement, la filière s'est entièrement disloquée: les producteurs de coton vendent exclusivement leur récolte à l'État, les tisserands laissent leurs métiers, et les fileuses, elles aussi, semblent abandonner leur poste.

Compte tenu de ces circonstances particulières, les enquêtes ne pouvaient s'ancrer dans la pratique mais étaient, au contraire, cantonnées au registre de la mémoire. L'enjeu du projet n'était plus uniquement documentaire, il devenait aussi méthodologique. Comment rendre compte d'activités techniques disparues? Une étude technologique est-elle possible uniquement sur la base de la mémoire des praticiens? Quelles sont les modalités ou les vecteurs de la mémoire technique? etc. Nous proposons, dans cet article, une piste de réflexion émergée au fur et à mesure des progrès du terrain. En détaillant une série de rencontres avec d'anciennes fileuses de coton, nous verrons que les situations d'enquête doivent être considérées comme des éléments d'analyse pertinents pour une ethnographie d'une technique disparue. 


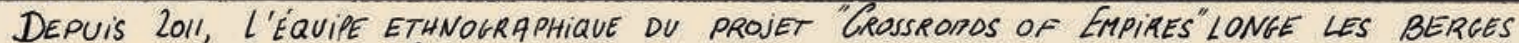
BENINOISES DU FLEUVE NIGER.

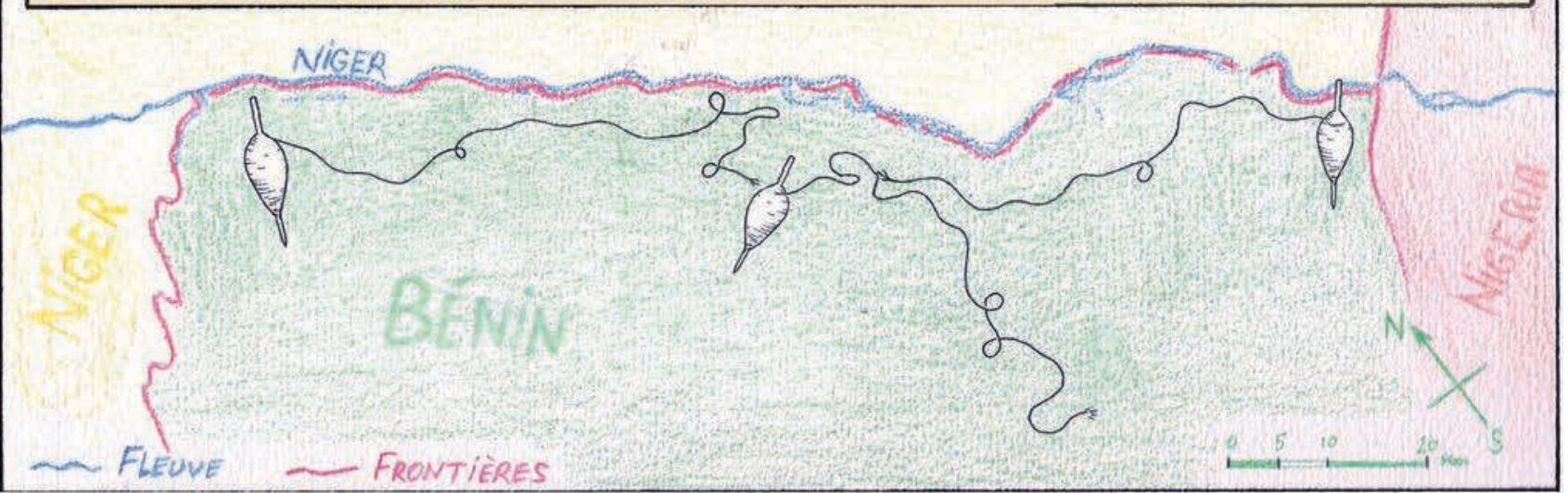

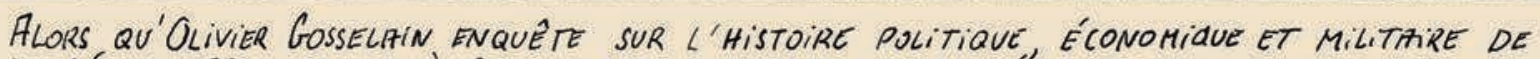
LA RÉCION, JE CHER LHE A' RENCONTRER LES DERNIERS REPRÉSENTANTS DE LA FILIERE textile artisanale dU DENDi (abandonneE dePUis plus de TRENTE ans).
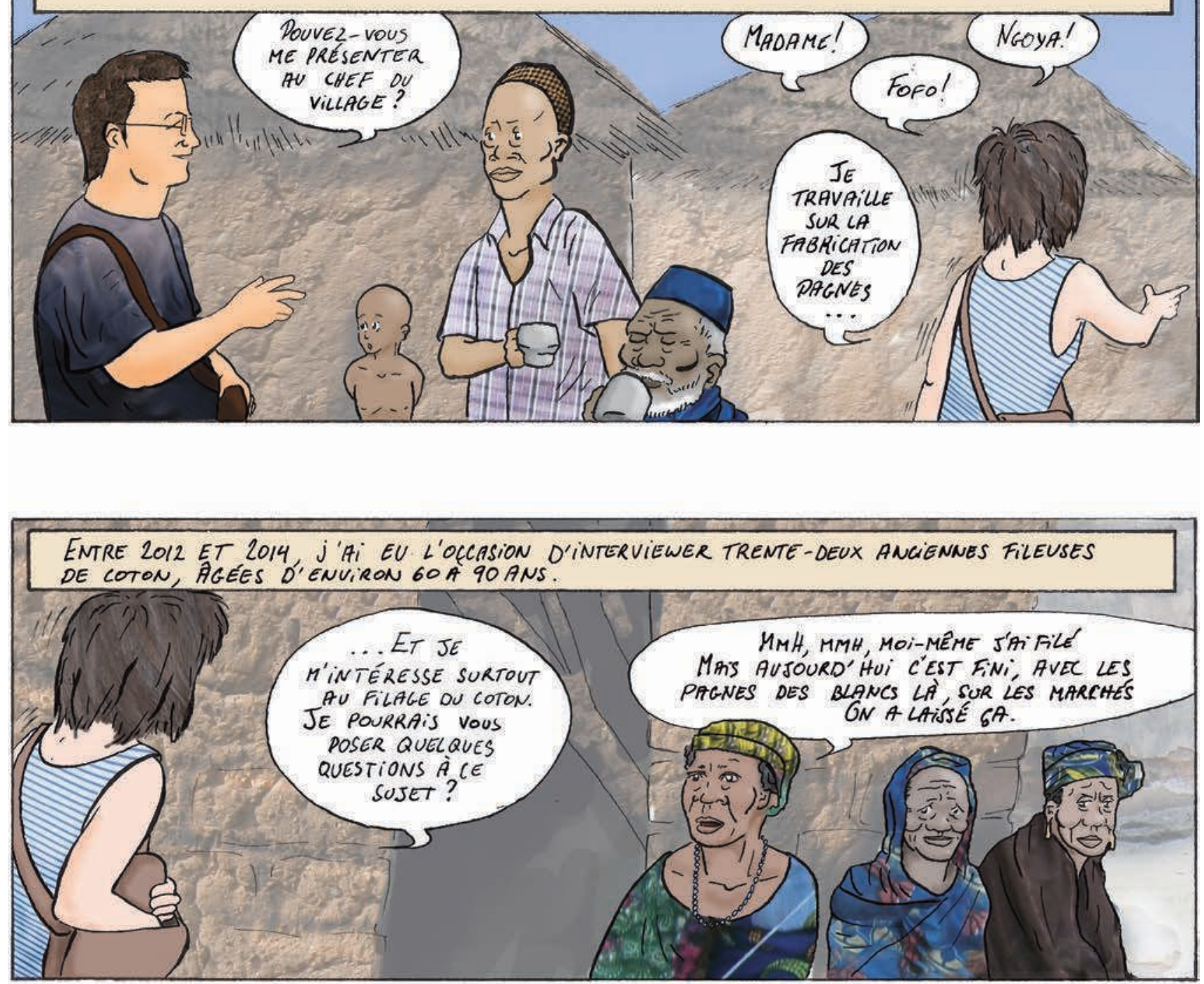

Lucie Smolderen \& Romain Minguet 

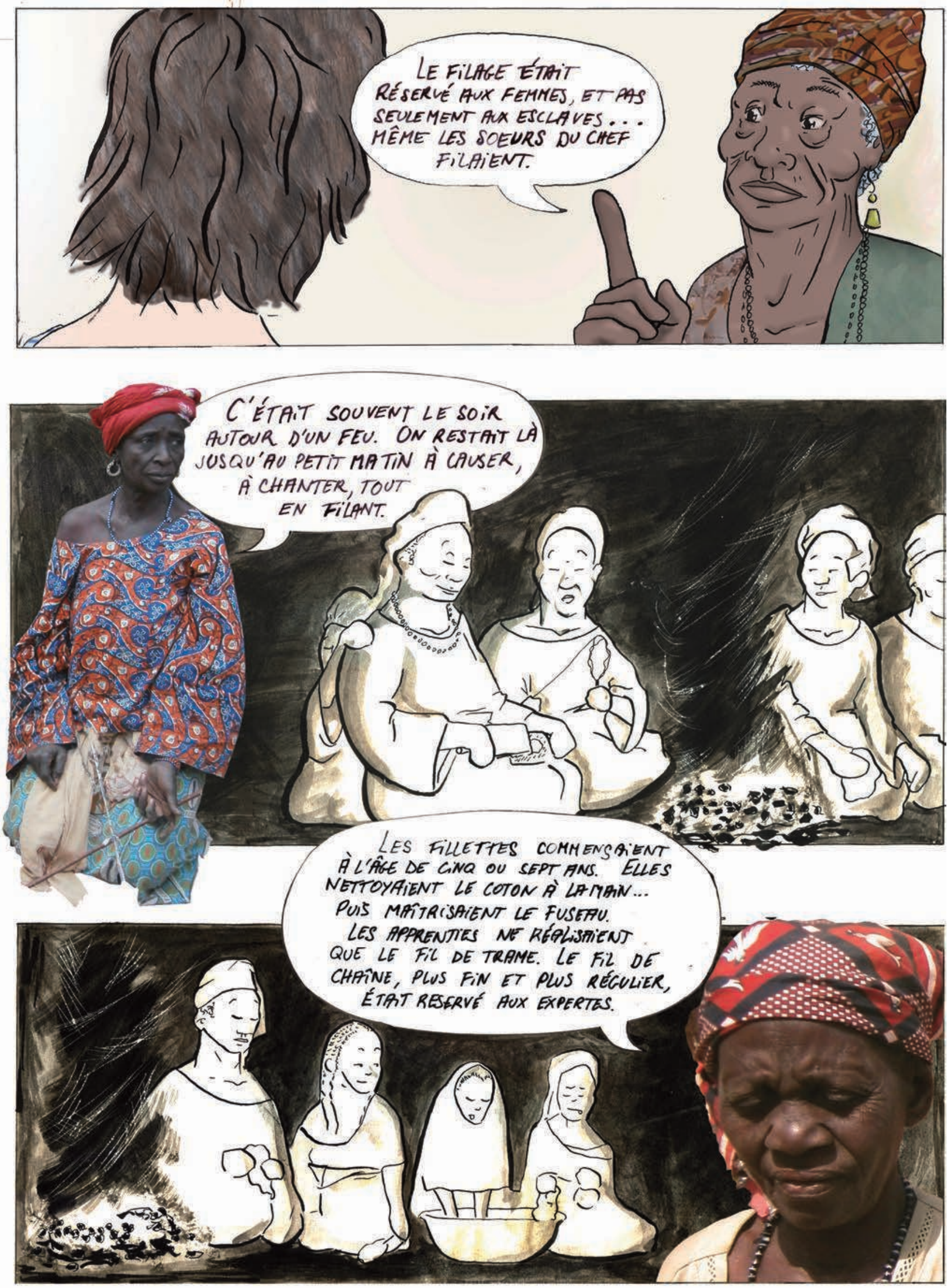

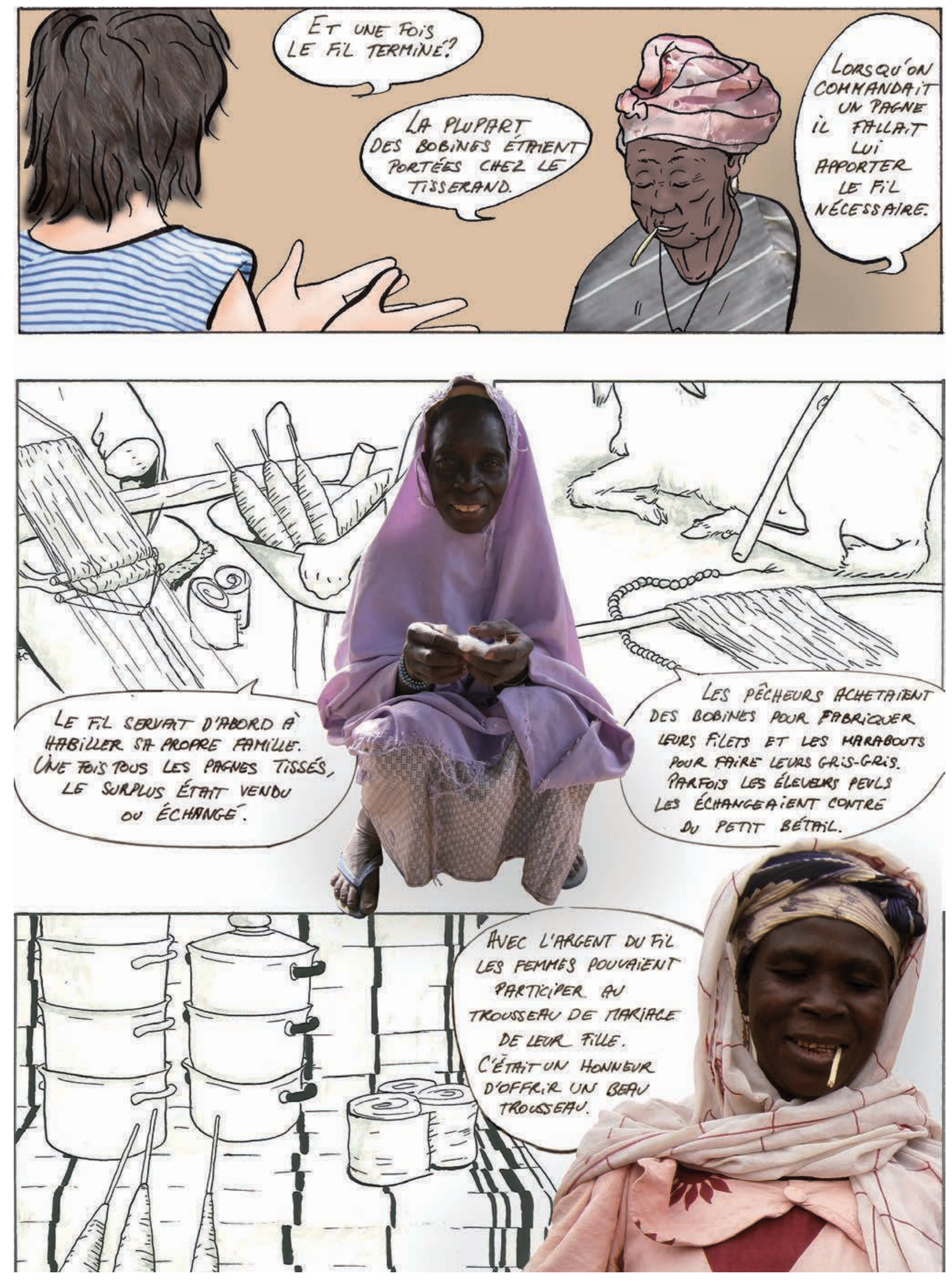

308 Lucie Smolderen \& Romain Minguet 

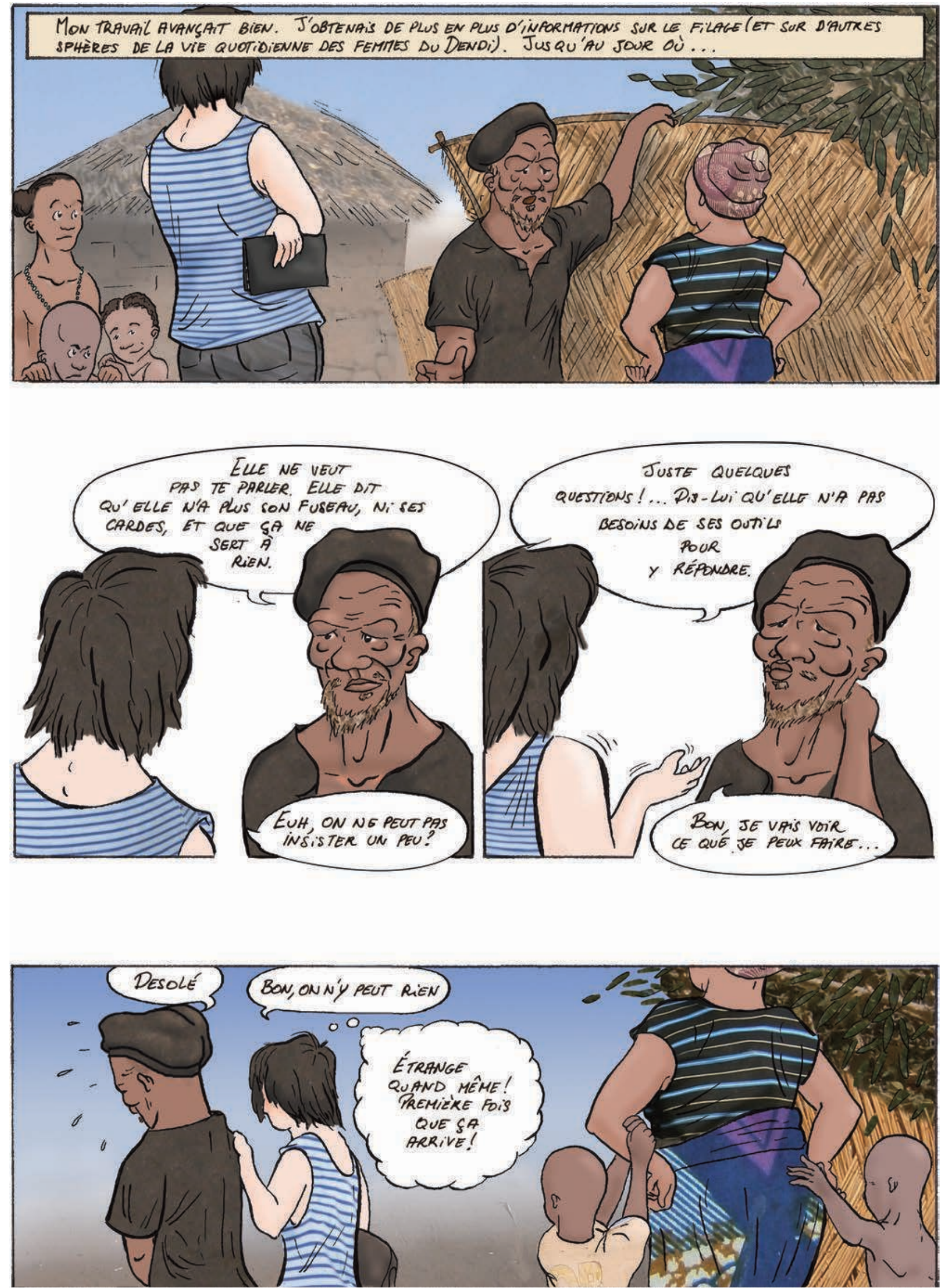
Lette rencontre (manquée) m'avait laissée songeuse... Pourquoi cette femme n'avait-elle pas voulu m'adresser la parole? La présence des objets était-elle à ce point indispensable?

La filière que j'étudiais était abandonnée, plus personne ne tissait, plus personne ne filait. Consciente de cet état de fait, je m'étais concentrée sur linterview des témoins dont les réminiscences constituaient, jen étais sûre, la seule source capable de m'éclairer sur cette filière. La mémoire de mes interlocutrices m'apparaissait comme un réservoir de récits à enregistrer. La dimension technique (ainsi que les objets qui lui sont associés) avait disparu de mon étude: le discours des informateurs représentait linnique canal utile pour "cette ethnographie différée", qui visait à reconstituer une tech. nique disparue. Pourtant, javais souvent en loccasion de voir en action les mains ridées et agiles de mes interlocutrices...

focalisée sur les paroles, j'avais oublié de prêter attention aux composantes concrètes de lenvironnement dans lequel se déroulait les enquêtes.

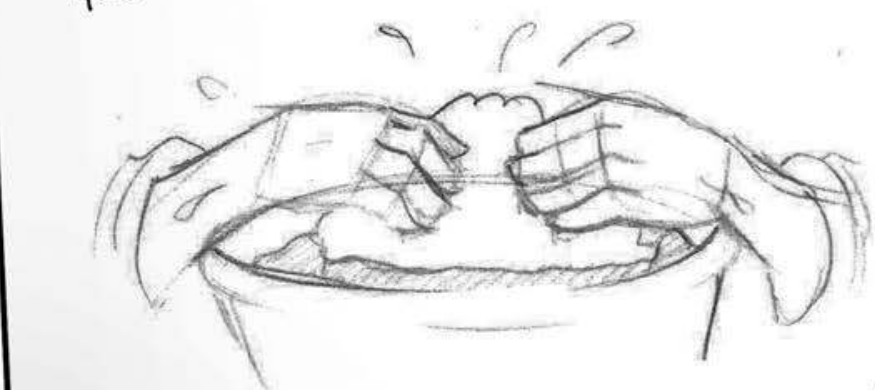

Nettoyage

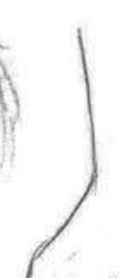

$c^{\prime}$

ge

m'

cu

ci

so

di

SI

$d$

S

5

S

$x$

e

1
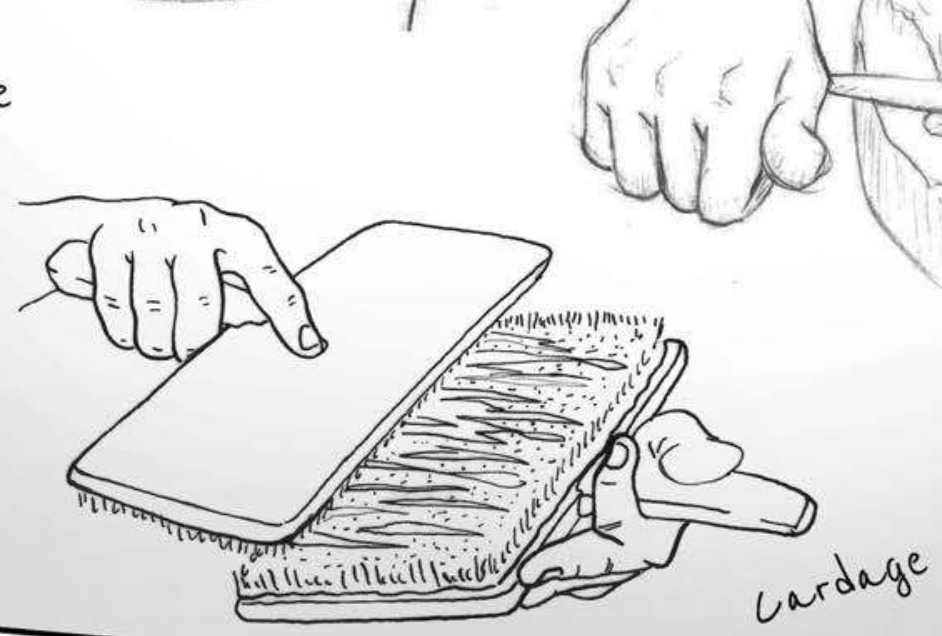

Egrenage.

310

Lucie Smolderen \& Romain Minguet 
C'est de retour à mon bureau que lomniprésence des objets et des gestes qui avaient accompagné tous les entretiens commençait à m'intriguer. Les actrices elles-mêmes avaient fait resurgir la culture matérielle et la technique. Comme par réflexe, ces anciennes fileuses se replongeaient dans l'action. Elles se prêtaient sous mes feux à une reprise jouée (un (k re-enactment $\|)$ - sorte de "reconstritution" - du filage dans laquelle l'acte technique refaisait surface. Une petrte poignée de coton (mise de côté lors de la dernière récolte), un sac plastique troué contenant quelques fu. seaux ou encore une paire de cardes aux clous tordus ressortaient spontanément. Parfois, il ne s'agissait que de me les montrer mais souvent ils étaient mis en action par les gestes précis de ces pra. xiciennes. De ce constat découlait une série de questions... Pouvais-je encore prétendre que cette activité avait disparu, alors que j’avais

n- pul'observer en acte? Cette reconstitution avait-elle le même

$n-$ statut que l'acte technique d'origine? Quid du potentiel heuristique de ces gestes et de ces objets? Devais-je les considérer avec l'xil de l'ethnologue?... Celui de l'archéologue? Ces questions sont toujours en suspens. Mais en vue d'y répondre, jai tenté de décrire différentes situations d'enquêtes au cours desquelles le filage a retrouvé sa matérialité...


xordage

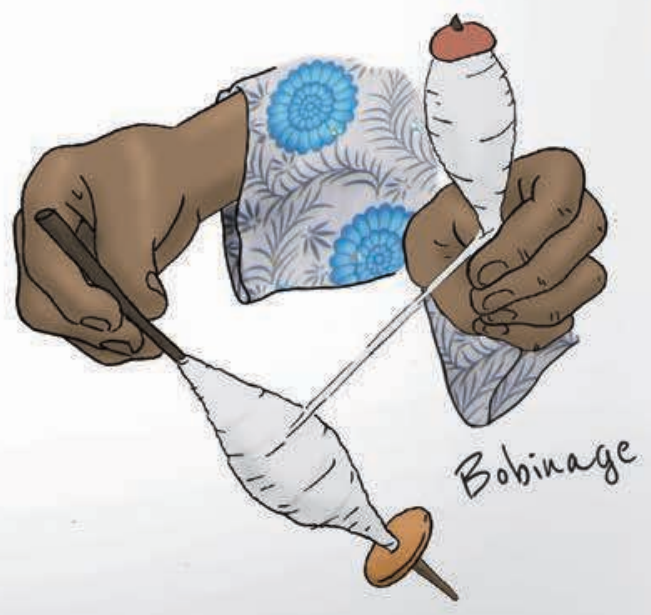



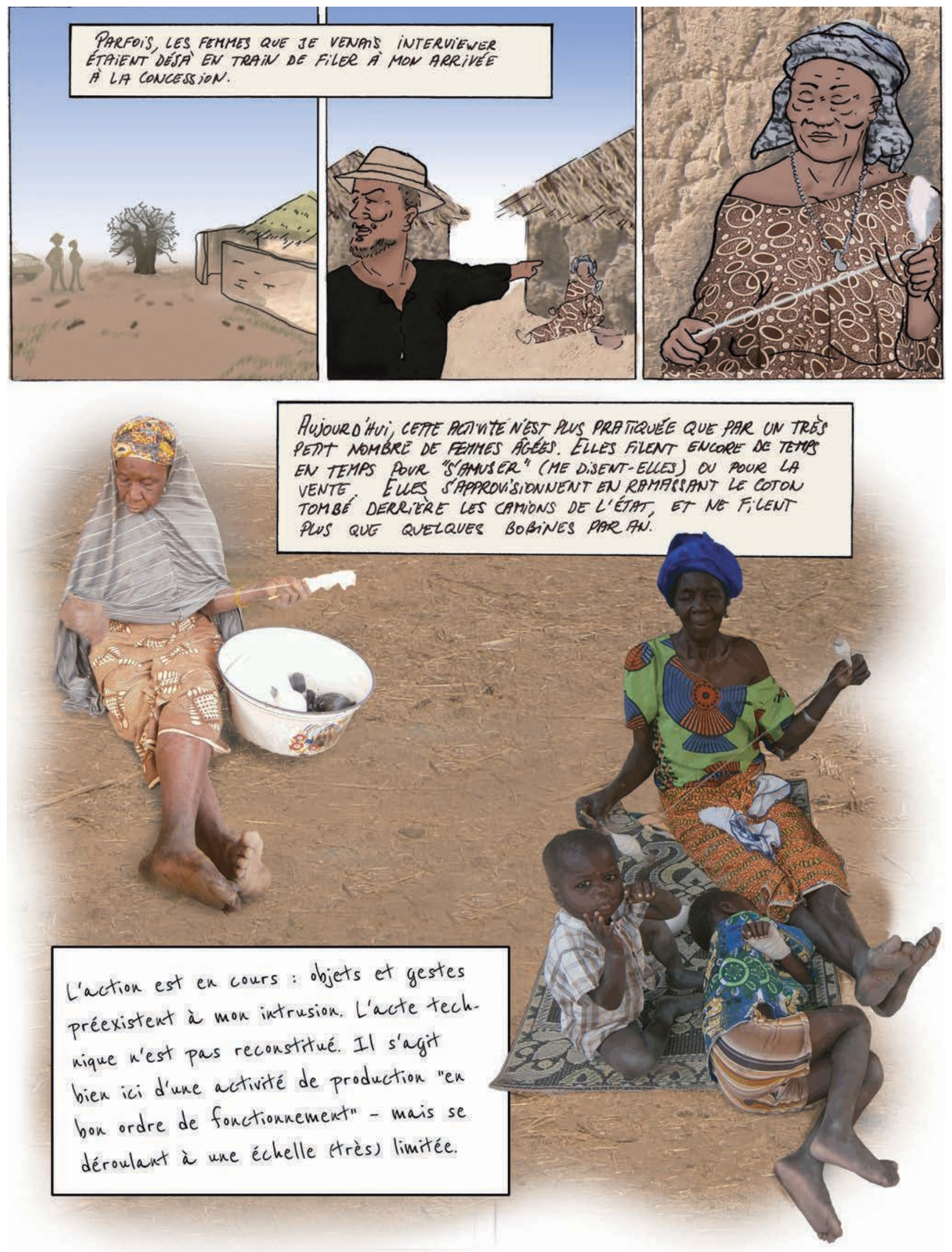
EN GÉNǴRAL, LES INFORMATRICES N'AURTIENT PLUS EN LEUR POSSESSION L'ENSEMBLE DES OUTILS NÉCESSAIRES AU DÉVELOPPEMENT DE LA CHAINE OPERATOIRE. EULS ENVOYAIENT UN ENFANT LES EMPRUNTER A UNE VOISINE.
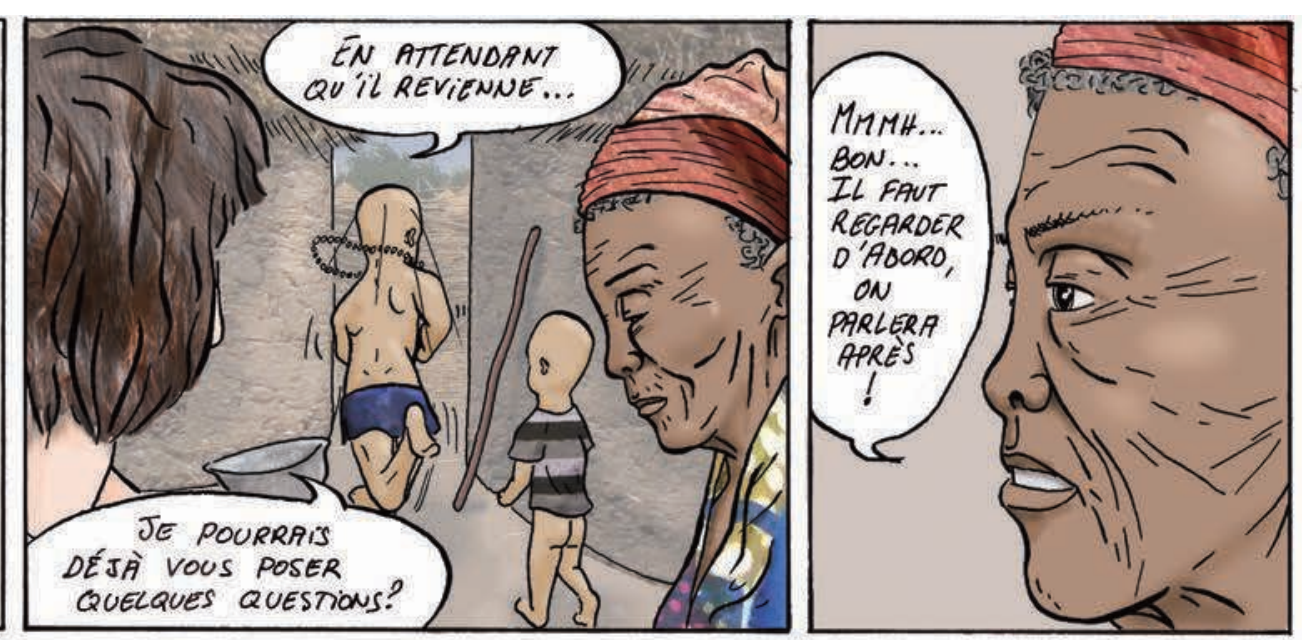

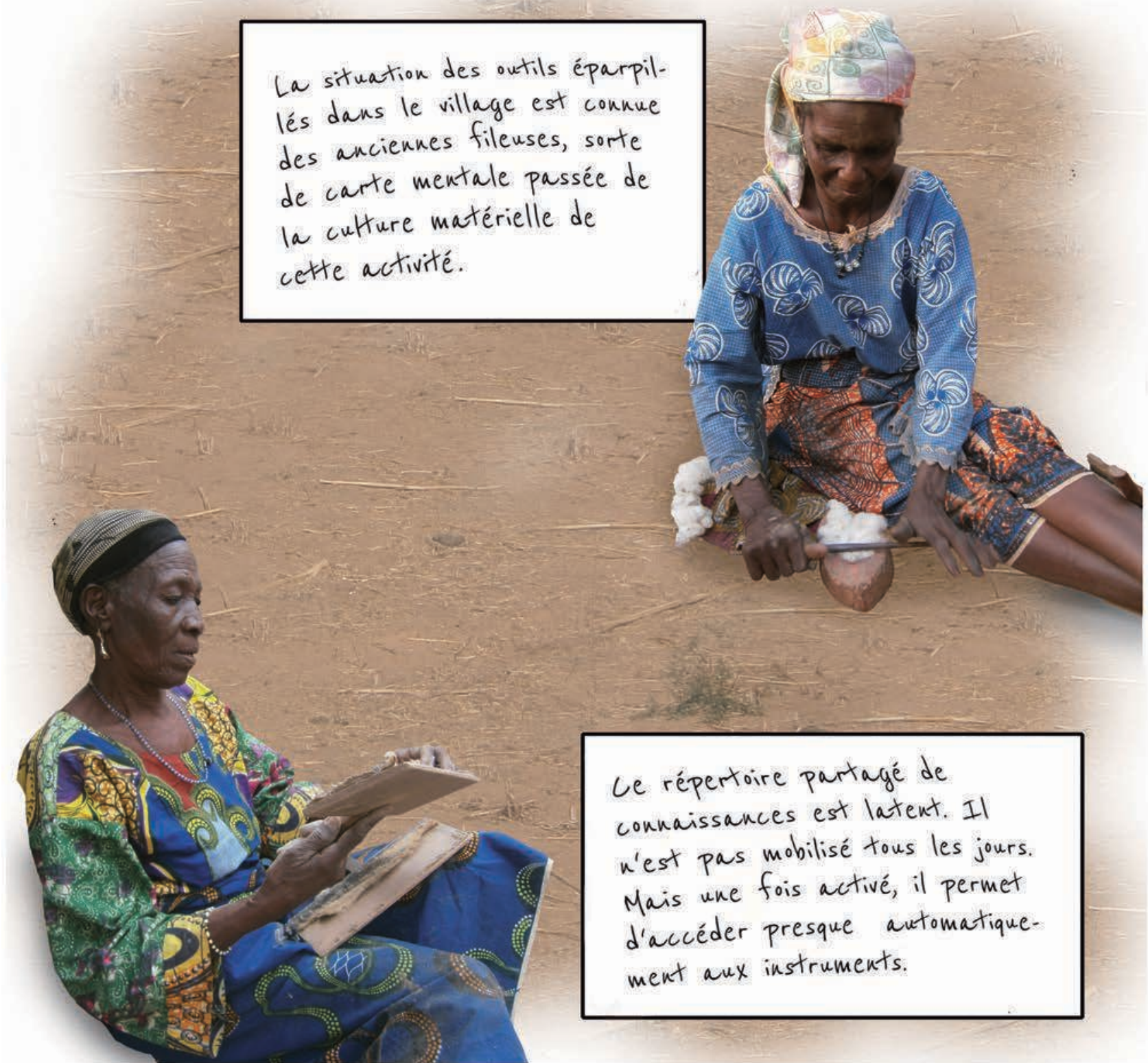




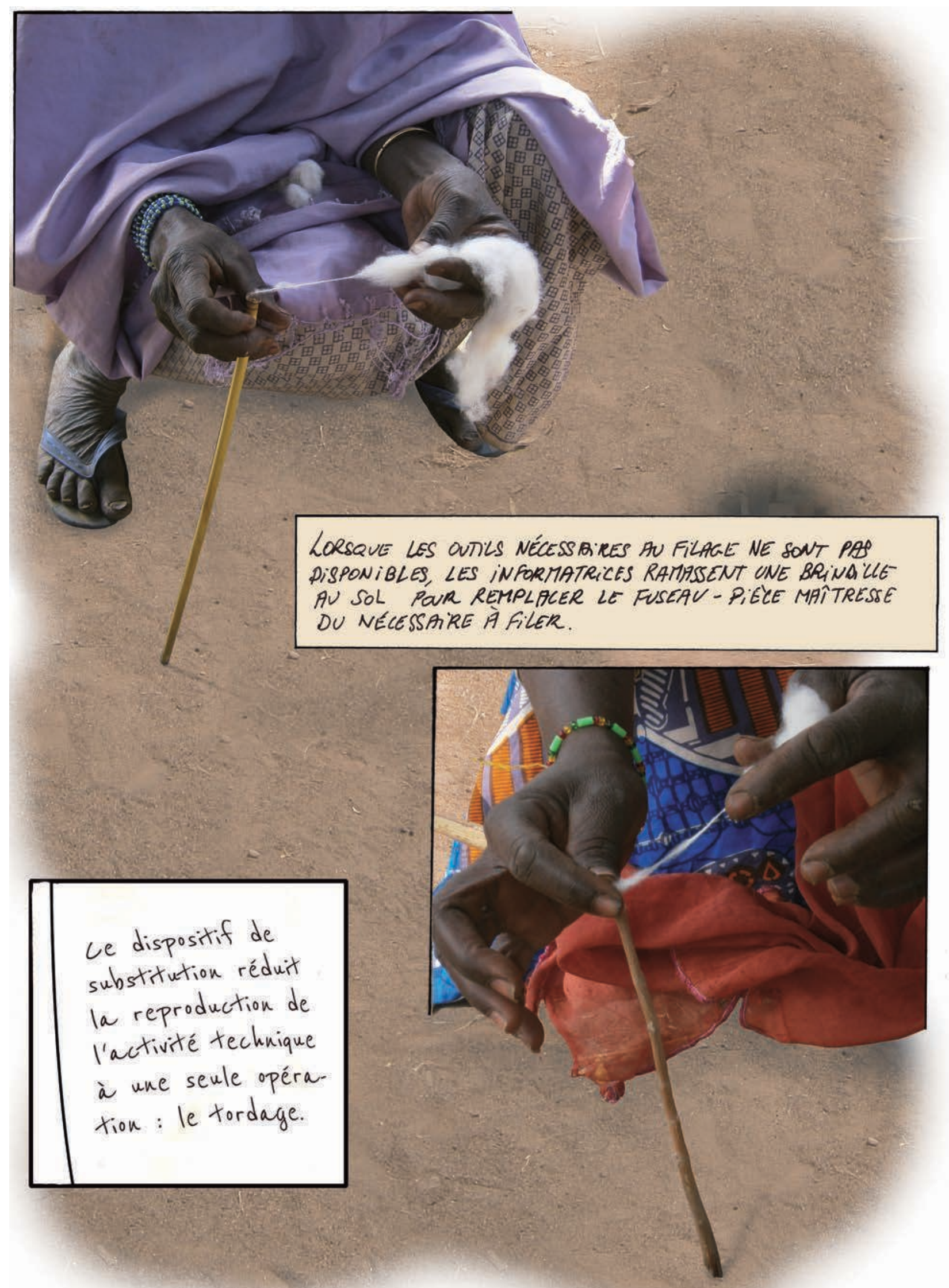


D'après les témoignages livrés par mes informatrices, le filage constituait (par le passé) l'une des activités quotidiennes principales de production textile, cette activité s'est progressivement vue don de la filière artisanale de production textilage n'appartient plus aujourd'hui qu'aux reléguée aux marges de l'univers social Lénation précédente. L'acte technique ne subsiste dernières représentantes de la génération préeeddente. L'ace ces restes de lactivité de filage cobservé qu'à l'état de trace... À première les entretiens) peuvent être assimiles a des vestiges (quasis archéologiques. et, une analyse technologique. La description des situations d'enquêtes ajoute cependant une autre dimension: pris en situation, ces gestes et ces objets deviennent des points d'ancrage pertinents dans la reconstitution du monde social des d'éclairer cette nouvelle dimension, une attention particulière aux modalités selon les. quelles ces vestiges out été convoqués lors de nos interviews est nécessaire.

Dans le premier type de situations rencontrées, mes interlocutrices ont conservé leur nécessaire à filer et filent encore de temps en temps. Le filage et ses objets, aupara. vant hérités de mère en fille et constitutifs de l'identité de jenne fille $s$ bonne à marier "1, sont aujourd'hui conservés précieusement dans la case d'une vieille femme. Dans le deuxième et troisième type de situations décrites - les plus courantes -, le surgissement des objets résulte d'une succession de médiations, certes stimulées par mon intrusion dans la concession, mais initiées par mes interlocutrices. A plusieurs reprises, la présence des objets est apparue comme une condition préalable à notre discussion. Deux séries de médiation doivent être distinguées. Dans le premier cas, l'interlocutrice convoque une carte mentale de la position des objets dans le village, réquisitionne un enfant dans le rôle du messager qui se rend alors chez une voisine pour quérir le matériel nécessaire. Le reconstitution peut alors commencer. Dans le second cas, l'interlocutrice s'appuie sur un dispositif de substitution: la chaine opératoire complète est mentalement réduite à l'opération centrale du tordage des fibres, un condensé de la chaine opératoire complète suffit à la reconstitution.

Les reconstitutions techniques ainsi que l'effort déployé par les interlocutrices pour les mettre en oeuvre cnotamment perceptible au travers de la multiplicité de modalités selon lesquelles les vestiges sont convoqués) transforment le statut des objets et des gestes. Ils ne sont plus uniquement des vestiges matériels de l'activité technique. Leur apparition et leur manipulation canalisent les remémorations. Ils offrent, dès lors, une prise pour le chercheur quitente de reconstituer un monde social disparu. 
LORS D'UNE DE MES RENCONTRES MON INTERLOCUTRICE SE MET A A CESTICULER. JERECONNASS LE GESTE ET LE SON DU fuseaU QVi TOURNOT. Elle MIME LE TORDAGE DES FiBRES LES TRALES D'UN GESTE TECHNIQVE PEUVENT S'AVERER INFINITESIMALES...

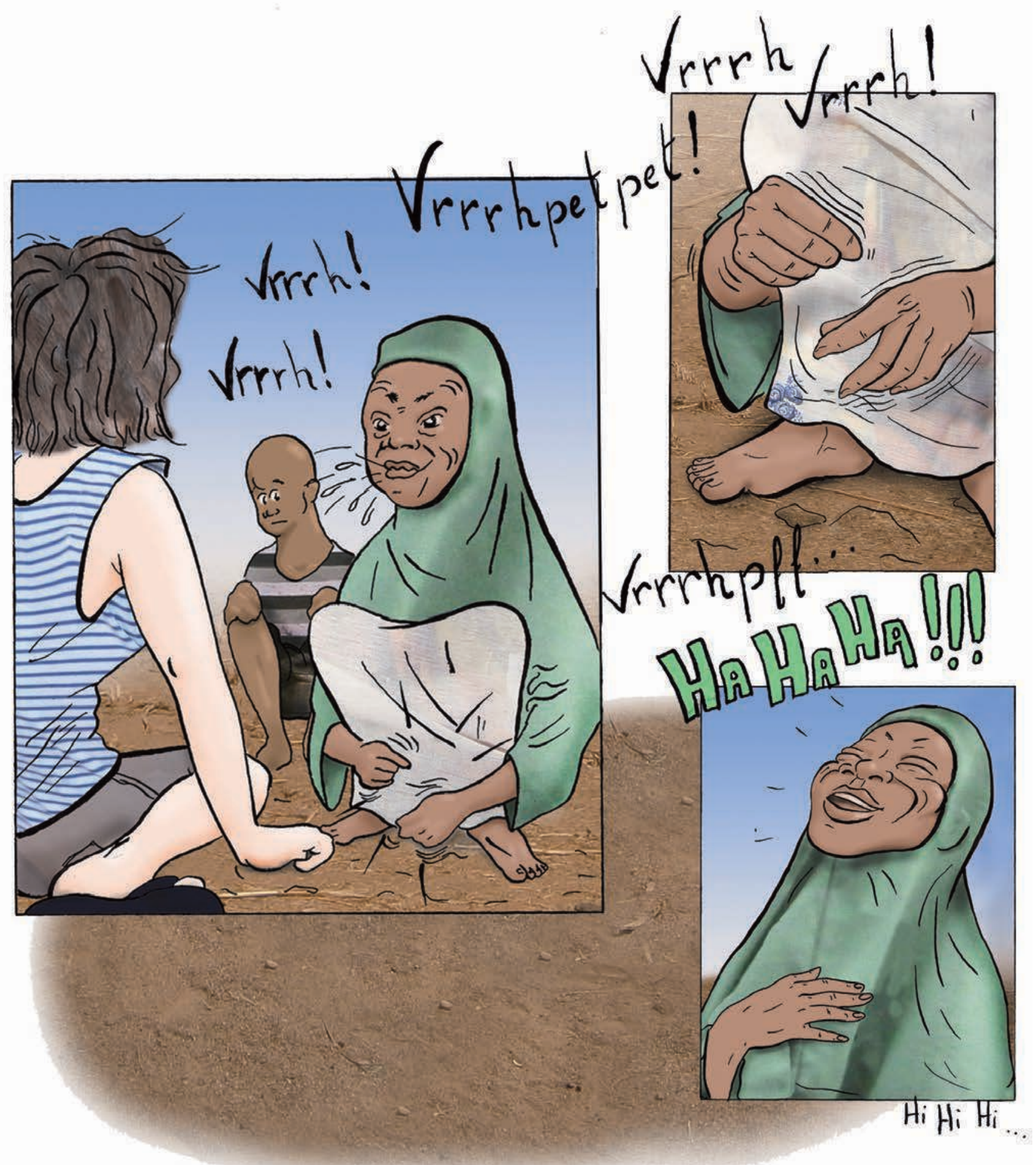




\section{AVEC L'AIMABLE PARTICIPATION DE}

La femme au nom inconnu qui a refusé l'interview, ville de Karimama

Alfari Adisa, née en 1925, ville de Karimama Dadé Ramatou, née en 1932, ville de Karimama Bawa (dit « Talou »), née vers 1952, ville de Karimama Azara Djahé, née vers 1945, ville de Karimama Raki Tahérou, née vers 1940, ville de Karimama Méri Adamou, née vers 1945, ville de Karimama Ayasatou (dit « Tana »), née vers 1957, village de Kwaratégui Boudjouie (dit « Sababe »), née vers 1932, village de Kwaratégui

Soumana Gambi, née en 1938, village de Bonwalou Salé Mémouna, née vers 1962, village de Pékinga Amina Salé, âge inconnu, village de Kompa Zenabu Messamaïri, née vers 1947, village de Kompa Kagoro Kadam, née vers 1962, village de Kompa Adisa Garba, née vers 1943, village de Kompa Azara Fonda, âge inconnu, village de Kompa
Ibounou Hadi, née vers 1960, village de Kompa Maryam Asawé, née vers 1963, village de Kompa Gambi (dit « Talou »), née vers 1925, village de Birnin Lafyia Maryam Souley, née vers 1932, village de Toumboutou Aïssa Gourza, âge incconu, village de Bogo Bogo Dere Kwarakwey, âge inconnu, village de Kantoro Boma Nati, née vers 1933, village de Loumbou Loumbou Nassanou Ramatou, née vers 1965, village de Bonyami Garba Adjo, née vers 1963, village de Bonyami Soumana Kadi, née vers 1958, village de Bonyami Hamani Adjo, née vers 1943, village de Bonyami Salé Adjo, née vers 1943, village de Bonyami Soumana Aïcha, née vers 1938, village de Bonyami Adamou Myriam, née vers 1937, village de Bonyami Hassan Safi, née vers 1934, village de Bonyami Fatoma Biobagu, née vers 1932, ville de Banikoara Bio Soumé Bérou, née vers 1962, village de Founougo

\section{ABSTRACT}

Ariane's thread in the Dendi (North Benin). Ethnography of an abandoned technique. In 2012, Lucie Smolderen started a study of the textile industry techniques in the Dendi region (North Benin). However, all activity in this domain had been abandoned forty years before. Direct observation of the techniques in their original context was thus impossible and she was obliged to resort to systematic interviews with ex-artisans in order to conduct her study. During these interviews, ex-spinners often proposed demonstrations of their former activity. Considering these technical reconstitutions - and the concrete circumstances in which the interviews themselves took place - led to a rethinking of her fieldwork methodology. The contextualized approach, as it is developed here, changes the epistemological status of the reconstitutions themselves. They are not mere demonstrations of the technical act, but become privileged channels of access to a global social environment that has disappeared. This article proposes to follow the development of these ideas, which like Ariane's thread guide us through the labyrinth of the fieldwork. Illustrator Romain Minguet's artwork contributes to a more global and immersive understanding of the complexity of the relationships between the observer and the observed, the present and the past, the technical and the social aspects of this type of study.

\section{MOTS-CLÉS}

Bénin, bande-dessinée ethnographique, récit graphique, technique disparue, filage, reconstitution, objet, situation d'enquête

\section{KEYWORDS}

Benin, ethnographic comics, graphic narrative, abandoned technique, spinning, reconstitution, object, fieldwork situation

\section{POUR CITER CET ARTICLE}

Smolderen, L. \& Minguet, R. 2014 Un fil d'Ariane dans le Dendi, in S. Boulay \& M.-L. Gélard, Vivre le sable! Corps, matière et sociétés, Techniques \& Culture 61 : 304-317. 